\title{
On Smooth Bicubic Surfaces from Quad Meshes
}

\author{
Jianhua Fan and Jörg Peters \\ Dept CISE, University of Florida
}

\begin{abstract}
Determining the least $m$ such that one $m \times m$ bi-cubic macropatch per quadrilateral offers enough degrees of freedom to construct a smooth surface by local operations regardless of the vertex valences is of fundamental interest; and it is of interest for computer graphics due to the impending ability of GPUs to adaptively evaluate polynomial patches at animation speeds.

We constructively show that $m=3$ suffices, show that $m=2$ is unlikely to always allow for a localized construction if each macro-patch is internally parametrically $C^{1}$ and that a single patch per quad is incompatible with a localized construction. We do not specify the GPU implementation.
\end{abstract}

\section{Introduction}

Quad(rilateral) meshes are used in computer graphics and CAD because they capture symmetries of natural and man-made objects. Smooth surfaces of degree bi-3 can be generated by applying subdivision to the quad mesh [CC78] or, alternatively, by joining a finite number of polynomial pieces [Pet00]. When quads form a checkerboard arrangement, we can interpret $4 \times 4$ grids of vertices as B-spline control points of a bi-cubic tensor product patch. Then we call the central quad ordinary and are guaranteed that adjacent ordinary quad patches join $C^{2}$.

The essential challenge comes from covering extraordinary quads, i.e. quads that have one or more vertices of valence $n \neq 4$ as illustrated in Fig. 1, left. While this can be addressed by recursive subdivision schemes, in many scenarios, for example GPU acceleration, localized parallel constructions of a finite number of patches are preferable $\left[\mathrm{NYM}^{+} 08\right]$. Here localized, parallel means that each construction step is parallel for all quads or vertices and only needs to access a fixed, small neighborhood of the quad or vertex. Due to the size limitations, this paper does not discuss GPU specifics, but addresses the fundamental lower bound question: how to convert each extraordinary quad into a macro-patch, consisting of $m \times m$ bi-cubic pieces, so as that a general quad mesh is converted into a smooth surface.

Prompted by the impending ability of GPUs to tessellate and adaptively evaluate finitely patched polynomial surface at animation speeds, there have recently been a number of publications close to this problem. Loop and Schaefer[LS08] propose bi-cubic $C^{0}$ surfaces with surrogate tangent patches to convey the impression of smoothness via lighting. Myles et al. [MYP08] perturb a bi-cubic 
base patch near non-4-valent vertices by coefficients of a $(5,5)$ patch to obtain a smooth surface. PCCM [Pet00] generates smooth bi-cubic surfaces but requires up to two steps of Catmull-Clark subdivision to separate non-4-valent vertices. This proves that $m=4$ suffices in principle. But bi-cubic PCCM can have poor shape for certain higher-order saddles (e.g. the 6-valent monkey saddle Fig. 5, row 3) as discussed in [Pet01]. Below we specify an algorithm that constructs smoothly connected $3 \times 3 C^{1}$ macro-patches without this shape problem; and discuss why the approach fails when $m<3$.
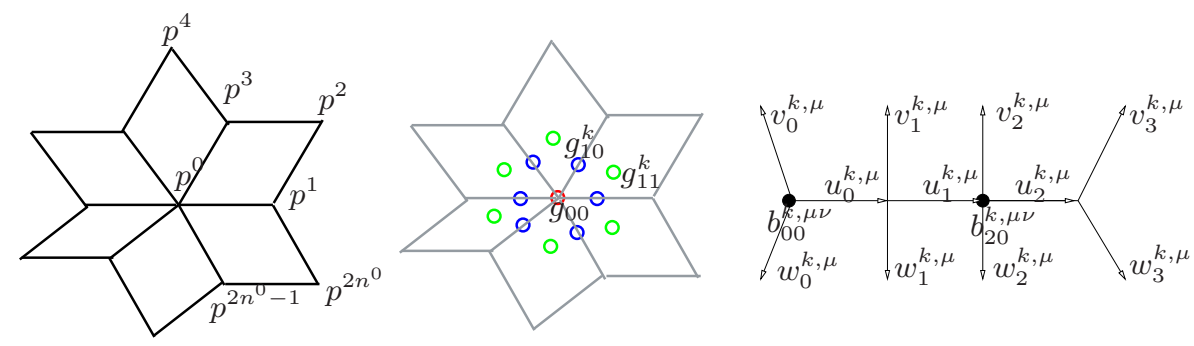

Fig. 1. left: extraordinary vertex $p^{0}$ with $n^{0}$ direct neighbors $p^{2 k-1}, k=1 \ldots n^{0}$. middle: limit point $g_{00}$, tangent points $g_{10}^{k}$ and 'twist' coefficients $g_{11}^{k}$. right: BB differences.

\section{Notation, and why $m=1$ need not be considered}

We denote the $k$ th bi-cubic Bernstein Bézier (BB) patch, $k=1 \ldots n^{0}$, surrounding a vertex $p^{0}$ of valence $n^{0}$ (Fig. 1) by

$$
\mathbf{b}^{k, \mu, \nu}(u, v):=\sum_{i=0}^{3} \sum_{j=0}^{3} b_{i j}^{k, \mu, \nu}\left(\begin{array}{l}
3 \\
i
\end{array}\right) u^{i}(1-u)^{3-i}\left(\begin{array}{l}
3 \\
j
\end{array}\right) v^{i}(1-v)^{3-j}
$$

Here $\mu, \nu$ indicate a piece of the $m \times m$ macro-patch (see Fig. 2, left, for $m=$ 3 ). The BB coefficients (control points) of the tensor-product patch $\mathbf{b}^{k, \mu, \nu}$ are therefore labeled by up to 5 indices when we need to be precise (Fig. 2):

$$
b_{i j}^{k, \mu, \nu} \in \mathbb{R}^{3}, \quad k=1 \ldots n^{0}, \quad \mu, \nu \in\{0, \ldots, m-1\}, \quad i, j \in\{0,1,2,3\} .
$$

For the two macro-patches meeting along the $k$ th boundary curve $\mathbf{b}^{k, \mu 0}(u, 0)=$ $\mathbf{b}^{k-1,0 \mu}(0, u), \mu=0, \ldots, m-1$, we want to enforce unbiased (logically symmetric) $G^{1}$ constraints

$$
\partial_{2} \mathbf{b}^{k, \mu 0}(u, 0)+\partial_{1} \mathbf{b}^{k-1,0 \mu}(0, u)=\alpha_{i}^{k}(u) \partial_{1} \mathbf{b}^{k, \mu 0}(u, 0), \quad i=0 \ldots, m-1,
$$

where each $\alpha_{i}^{k}$ is a rational, univariate scalar function and $\partial_{\ell}$ means differentiation with respect to the $\ell$ th argument. If $\alpha_{i}^{k}=0$, the constraints enforce 
(parametric) $C^{1}$ continuity. The polynomial equalities (3) hold for the $k$ th curve exactly when all $m \times n^{0}$ polynomial coefficients are equal. The coefficients are differences of the $\mathrm{BB}$ control points:

$$
v_{i}^{k, \mu}:=b_{i 1}^{k, \mu 0}-b_{i 0}^{k, \mu 0}, \quad w_{i}^{k, \mu}:=b_{1 i}^{k-1,0 \mu}-b_{0 i}^{k-1,0 \mu}, \quad u_{i}^{k, \mu}:=b_{i+1,0}^{k, \mu 0}-b_{i 0}^{k, \mu 0} .
$$

The differences need only have a single subscript since we consider curves (in $u$ ) and a simpler superscript since $\nu=0$. For example, if we choose $\alpha_{i}^{k}(u):=$ $\lambda_{i}^{k}(1-u)+\lambda_{i+1}^{k} u$, then (3) formally yields $4 m$ equations when $\mu=0, \ldots, m$ :

$$
\begin{array}{rlrl}
v_{0}^{k, \mu}+w_{0}^{k, \mu} & = & \lambda_{\mu}^{k} u_{0}^{k, \mu} \\
3\left(v_{1}^{k, \mu}+w_{1}^{k, \mu}\right) & = & 2 \lambda_{\mu}^{k} u_{1}^{k, \mu}+\lambda_{\mu+1}^{k} u_{0}^{k, \mu} \\
3\left(v_{2}^{k, \mu}+w_{2}^{k, \mu}\right) & = & \lambda_{\mu}^{k} u_{2}^{k, \mu}+2 \lambda_{\mu+1}^{k} u_{1}^{k, \mu} \\
v_{3}^{k, \mu}+w_{3}^{k, \mu}= & \lambda_{\mu+1}^{k} u_{2}^{k, \mu} .
\end{array}
$$

By definition, (7) $)_{\mu=i}=(4)_{\mu=i+1}$, i.e. constraint (7) when substituting $\mu=i$ is identical to constraint (4) for $\mu=i+1$.

We need not consider $m=1$, i.e. one bi-cubic patch per quad, since the vertex-enclosure constraint [Pet02, p.205] implies, for even $n^{0}>4$ that the normal curvatures and hence the coefficients $b_{20}^{k, 00}\left(\bullet\right.$ Fig. 1,right) of the $n^{0}$ curves emanating from $p^{0}$ are related for $k=1, \ldots, n^{0}$ : the normal component of their alternating sum $\sum_{k}(-1)^{k} b_{20}^{k, 00}$ must vanish. Since, for a bi-cubic patch, the control point $b_{20}^{k, 00}$ lies in the tangent plane of the $k$ th neighbor vertex (Fig. 1,right), the vertex's enclosure constraint constrains the neighboring tangent planes with respect to its tangent plane. Therefore, if we fix the degree of the patches to be bi-cubic and allow only one patch per quad then no localized construction is possible.

For $m>1$, the coefficients $b_{20}^{k, 00}$ no longer lie in the tangent plane of the neighbor; so a local construction may be possible. We next give an explicit construction when $m=3$.

\section{Localized smooth surface construction using a $3 \times 3$ macro-patch}

We factor the algorithm into four localized stages. First, we define the central point $g_{00}$, the tangents $g_{10}-g_{00}$ and the face coefficients $g_{11}$ as an average (see Fig. 1) of

- the extraordinary vertex $p^{0}$ with valence $n^{0}$, and

- its 1-ring neighbors $p^{1}, p^{2}, \ldots, p^{2 n^{0}}$.

In a second stage, we partition the quad into a $3 \times 3$ arrangement (Fig. 2) and establish its boundary; in the third, we determine the cross-boundary derivatives so that pairs of macro-patches join $G^{1}$ (Equation (3)) and in the final stage, we determine the interior coefficients. By this construction, a macro-patch joins at least parameterically $C^{1}$ with an unpartitioned spline patch (see Fig. 5, row 2, where the second entry displays each polynomial piece in a different color). 
1. [Initialization] It is convenient (and shown to be effective to approximate the Catmull-Clark limit surface) to set $g_{i j}$ according to [MYP08]. That is to set $g_{00}$ to the limit of $p^{0}$ under Catmull-Clark subdivision (red circle in Fig. 1 middle) and place the $g_{10}^{k}$ (blue circle in Fig. 1 middle) on the Catmull-Clark tangent plane:

$$
\begin{aligned}
& g_{00}=g_{00}^{k}:=\frac{\sum_{l=1}^{n^{0}}\left(n^{0} p_{0}+4 p_{2 l-1}+p_{2 l}\right)}{n^{0}\left(n^{0}+5\right)}, \quad k=1 \ldots n^{0}, \\
& g_{10}^{k}:=g_{00}^{k}+e_{1} c_{n^{0}}^{k}+e_{2} s_{n^{0}}^{k}, \quad e_{i}:=\frac{\sigma_{n^{0}}}{3\left(2+\omega_{n^{0}}\right)} \sum_{j=1}^{n^{0}}\left(\alpha_{i} p^{2 j-1}+\beta_{i} p^{2 j}\right), \\
& g_{11}^{k}:=\frac{1}{9}\left(4 p^{0}+2\left(p^{2 k+1}+p^{2 k+3}\right)+p^{2 k+2}\right), \quad k=1 \ldots n^{0} .
\end{aligned}
$$

where the scalar weights are defined as

$$
\begin{aligned}
& c_{n^{0}}^{k}:=\cos \frac{2 \pi k}{n^{0}}, \quad s_{n^{0}}^{k}:=\sin \frac{2 \pi k}{n^{0}}, \quad c_{n^{0}}:=c_{n^{0}}^{1}, \\
& \omega_{n^{0}}:=16 \lambda_{n^{0}}-4, \quad \lambda_{n^{0}}:=\frac{1}{16}\left(c_{n^{0}}+5+\sqrt{\left(c_{n^{0}}+9\right)\left(c_{n^{0}}+1\right)}\right), \sigma_{n^{0}}:=\left\{\begin{array}{cc}
0.53 & \text { if } n^{0}=3, \\
\frac{1}{4 \lambda_{n^{0}}} & \text { if } n^{0}>3,
\end{array}\right. \\
& \alpha_{1}:=\omega_{n^{0}} c_{n^{0}}^{j-1}, \quad \beta_{1}:=c_{n^{0}}^{j-1}+c_{n^{0}}^{j}, \quad \alpha_{2}:=\omega_{n^{0}} s_{n^{0}}^{j-1}, \quad \beta_{2}:=s_{n^{0}}^{j-1}+s_{n^{0}}^{j} .
\end{aligned}
$$

Symmetric construction of the other three corners of the quad yields $4 \times 4$ coefficients $g_{i j}$ that can be interpreted as the BB coefficients of one bi-cubic patch $g:[0,1]^{2} \rightarrow \mathbb{R}^{3}$ in the form (1).
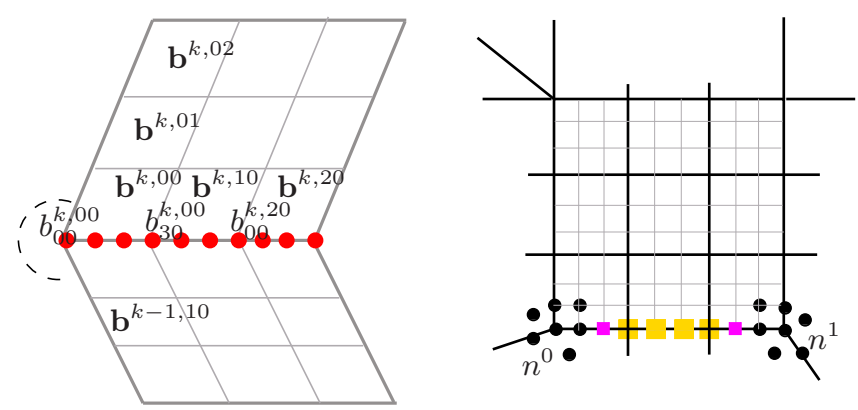

Fig. 2. left: Patches $\mathbf{b}^{k, \mu \nu}$ and coefficients $b_{i j}^{k, \mu \nu}$. right: Coefficients shown as black disks are determined by subdividing the initialization $g_{i j}$ (see (11)), coefficients shown as small squares are determined by (14) and (15), coefficients shown as big yellow squares are determined by $C^{2}$ continuity of the boundary curve (16), (17), (18).

2. [domain partition and boundary] We partition the domain into $3 \times 3$ pieces (see Fig. 2 left) and let the $3 \times 3$ macro-patch inherit vertex position and 
tangents (black disks in Fig. 2 right) by subdividing $g$ :

$$
b_{00}^{k, 00}:=g_{00}^{k}, \quad b_{10}^{k, 00}:=\frac{2}{3} g_{00}^{k}+\frac{1}{3} g_{10}^{k}, \quad b_{11}^{k, 00}:=\frac{4 g_{00}^{k}+2 g_{10}^{k}+2 g_{01}^{k}+g_{11}^{k}}{9} .
$$

Each macro-patch will be parametrically $C^{1}$ (and $C^{2}$ in the interior). To enforce the $G^{1}$ constraints (3) between macro-patches, we need to distinguish two cases when choosing $\alpha_{i}^{k}$ depending on whether one of the vertices is regular, i.e. has valence 4 . Of course, if both valences are 4 then we simply subdivide $g$ and set $\alpha_{i}^{k}=0$; and if all four corner points have valence 4, no partition is needed in the first place since $g$ is then part of a bi-cubic tensor-product B-spline patch complex and therefore joins $C^{2}$ with its spline neighbors and at least $C^{1}$ with macro-patches (Fig. 5, row 2, second entry).

2a. [boundary $n^{0} \neq 4 \neq n^{1}$ ] We choose $\alpha_{i}^{k}$ as simple as possible, namely linear

$$
\begin{gathered}
\text { if } n^{0} \neq 4 \neq n^{1}: \quad \alpha_{i}^{k}(u):=\lambda_{i}^{k}(1-u)+\lambda_{i+1}^{k} u, \\
\lambda_{0}^{k}:=2 \cos \left(\frac{2 \pi}{n^{0}}\right), \quad \lambda_{3}^{k}:=-2 \cos \left(\frac{2 \pi}{n^{1}}\right), \quad \lambda_{1}^{k}:=\frac{2 \lambda_{0}^{k}+\lambda_{3}^{k}}{3}, \lambda_{2}^{k}:=\frac{\lambda_{0}^{k}+2 \lambda_{3}^{k}}{3} .
\end{gathered}
$$

The three cubic pieces of the boundary curve have enough free parameters to enforce Equation (5) for $\mu=0$ and Equation (6) for $\mu=2$ and (small squares in Fig. 2 right) by setting

$$
\begin{aligned}
& b_{20}^{k, 00}:=b_{10}^{k, 00}+\frac{3\left(b_{11}^{k, 00}+b_{11}^{k-1,00}-2 b_{10}^{k, 00}\right)-\lambda_{1}^{k}\left(b_{10}^{k, 00}-b_{00}^{k, 00}\right)}{2 \lambda_{0}^{k}}, \\
& b_{10}^{k, 20}:=b_{20}^{k, 20}+\frac{\lambda_{2}^{k}\left(b_{30}^{k, 20}-b_{20}^{k, 20}\right)-3\left(b_{21}^{k, 20}+b_{12}^{k-1,02}-2 b_{20}^{k, 20}\right)}{2 \lambda_{3}^{k}}
\end{aligned}
$$

and joining the three curve segments $C^{2}$ (cf. large squares in Fig. 2 right)

$$
\text { for } \begin{aligned}
\mu=0,1: \quad b_{30}^{k, \mu 0} & :=\left(b_{20}^{k, \mu 0}+b_{10}^{k, \mu+1,0}\right) / 2, \\
b_{10}^{k, 10} & :=\frac{4}{3} b_{20}^{k, 00}-\frac{1}{3} b_{20}^{k, 20}+\frac{2}{3} b_{10}^{k, 20}-\frac{2}{3} b_{10}^{k, 00}, \\
b_{20}^{k, 10} & :=\frac{2}{3} b_{20}^{k, 00}-\frac{2}{3} b_{20}^{k, 20}+\frac{4}{3} b_{10}^{k, 20}-\frac{1}{3} b_{10}^{k, 00} .
\end{aligned}
$$

2b. [boundary $n^{0} \neq 4=n^{1}$ ] If $n^{0} \neq 4=n^{1}$, Lemma 2 in the Appendix shows that we cannot choose all $\alpha_{i}^{k}$ to be linear. (If $\lambda_{1}^{k}=0$ in Lemma 2 then the dependence appears at the next 4 -valent $\operatorname{crossing} b_{00}^{k, 10}$.) We set $\lambda:=2 \cos \left(\frac{2 \pi}{n^{0}}\right)$ and

$$
\alpha_{0}^{k}(u):=\lambda^{k}(1-u)+\frac{\lambda^{k}}{2} u, \quad \alpha_{1}^{k}(u):=\frac{\lambda^{k}}{2}(1-u)^{2}, \quad \alpha_{2}^{k}(u)=0 .
$$

Then $b_{20}^{k, 00}$ is defined by (5) and $b_{10}^{k, 20}$ by subdividing the cubic boundary of $g$ :

$$
b_{10}^{k, 20}:=-\frac{2}{9} b_{00}^{k, 00}+\frac{1}{3} b_{10}^{k, 00}+\frac{4}{3} b_{20}^{k, 20}-\frac{4}{9} b_{30}^{k, 20} .
$$


From the remaining six $G^{1}$ constraints across the macro-patch boundary,

$$
\begin{aligned}
3\left(v_{2}^{k, 0}+w_{2}^{k, 0}\right) & =\lambda^{k} u_{2}^{k, 0}+\lambda^{k} u_{1}^{k, 0}, & 3\left(v_{2}^{k, 1}+w_{2}^{k, 1}\right) & =0 \\
3\left(v_{3}^{k, 0}+w_{3}^{k, 0}\right) & =\frac{3}{2} \lambda^{k}\left(b_{10}^{k, 10}-b_{00}^{k, 10}\right), & 3\left(v_{3}^{k, 1}+w_{3}^{k, 1}\right) & =0 \\
9\left(v_{1}^{k, 1}+w_{1}^{k, 1}\right) & =\frac{3}{2} \lambda^{k}\left(b_{30}^{k, 10}-b_{20}^{k, 10}\right), & 9\left(v_{1}^{k, 2}+w_{1}^{k, 2}\right) & =0 .
\end{aligned}
$$

the two listed as (22) are linked to the remaining four by the requirement that the macro-patches be internally $C^{1}$ :

$$
\text { for } \begin{aligned}
\mu=0,1: \quad b_{30}^{k, \mu 0} & =\left(b_{20}^{k, \mu 0}+b_{10}^{\mu+1,20}\right) / 2 \\
v_{2}^{k, \mu}+v_{1}^{k, \mu+1} & =2 v_{3}^{k, \mu}, \quad w_{2}^{k, \mu}+w_{1}^{k, \mu+1}=2 w_{3}^{k, \mu} .
\end{aligned}
$$

Thus, by adding 3 times (21) to (23) and subtracting 6 times (22) and observing (25), we eliminate the left hand sides and obtain one constraint purely in the boundary coefficients multiplied by $\lambda^{k} \neq 0$. A second constraint arises since $\alpha_{1}^{k}(u)$ being quadratic implies that the middle segment $\mathbf{b}^{k, 10}(u, 0)$ is quadratic, i.e. its third derivative is zero:

$$
b_{30}^{k, 10}-3 b_{20}^{k, 10}+3 b_{10}^{k, 10}-b_{00}^{k, 10}=0 .
$$

Both constraints are enforced by setting

$$
b_{10}^{k, 10}:=\frac{41}{25} b_{20}^{k, 00}+\frac{4}{25} b_{10}^{k, 20}-\frac{4}{5} b_{10}^{k, 00}, \quad b_{20}^{k, 10}:=\frac{36}{25} b_{20}^{k, 00}+\frac{9}{25} b_{10}^{k, 20}-\frac{4}{5} b_{10}^{k, 00} .
$$

Together with (24), this fixes the macro-patch boundary (Fig. 2, right).

3. [First interior layer, $G^{1}$ constraints] Enforcing the remaining four $G^{1}$ constraints in terms of the red coefficients in Fig. 3 is straightforward and our symmetric solution is written out below.

3a. $\left[n^{0} \neq 4 \neq n^{1}\right]$

$$
\begin{array}{rlrl}
h_{1 \mu}:=b_{20}^{k, \mu 0}+\frac{\lambda_{\mu}^{k} u_{2}^{k, \mu}+2 \lambda_{\mu+1}^{k} u_{1}^{k, \mu}}{6}, & h_{2 \mu}:=b_{10}^{k, \mu 0}+\frac{2 \lambda_{\mu}^{k} u_{1}^{k, \mu}+\lambda_{\mu+1}^{k} u_{0}^{k, \mu}}{6} \\
\mu=0,1: \quad b_{21}^{k, \mu 0}:=h_{1 \mu}+\frac{1}{2}\left(\tilde{b}_{21}^{k, 00}-\tilde{b}_{12}^{k-1,00}\right), & b_{12}^{k-1,0 \mu}:=h_{1 \mu}+\frac{1}{2}\left(\tilde{b}_{12}^{k-1,00}-\tilde{b}_{21}^{k, 00}\right) \\
\mu=1,2: \quad b_{11}^{k, \mu 0}:=h_{2 \mu}+\frac{1}{2}\left(\tilde{b}_{11}^{k, 10}-\tilde{b}_{11}^{k-1,01}\right), & b_{11}^{k-1,0 \mu}:=h_{2 \mu}+\frac{1}{2}\left(\tilde{b}_{11}^{k-1,01}-\tilde{b}_{11}^{k, 10}\right) . \\
\text { 3b. }\left[n^{0} \neq 4=n^{1}\right] h_{1}:=b_{20}^{k, 00}+\frac{\lambda_{0}^{k} u_{2}^{k, 0}+\lambda_{0}^{k} u_{1}^{k, 0}}{6}, \quad h_{2}:=b_{10}^{k, 10}+\frac{\lambda_{0}^{k} u_{2}^{k, 1}}{12} \\
b_{21}^{k, 00}:=h_{1}+\frac{1}{2}\left(\tilde{b}_{21}^{k, 00}-\tilde{b}_{12}^{k-1,00}\right), & b_{12}^{k-1,0 j}:=h_{1}+\frac{1}{2}\left(\tilde{b}_{12}^{k-1,00}-\tilde{b}_{21}^{k, 00}\right) \\
b_{11}^{k, 10}:=h_{2}+\frac{1}{2}\left(\tilde{b}_{11}^{k, 10}-\tilde{b}_{11}^{k-1,01}\right), & b_{11}^{k-1,01}:=h_{2}+\frac{1}{2}\left(\tilde{b}_{11}^{k-1,01}-\tilde{b}_{11}^{k, 10}\right) \\
b_{21}^{k, 10}:=b_{20}^{k, 10}+\frac{1}{2}\left(\tilde{b}_{21}^{k, 10}-\tilde{b}_{12}^{k-1,01}\right), & b_{12}^{k-1,01}:=b_{20}^{k, 10}+\frac{1}{2}\left(\tilde{b}_{12}^{k-1,01}-\tilde{b}_{21}^{k, 10}\right) \\
b_{11}^{k, 20}:=b_{10}^{k, 20}+\frac{1}{2}\left(\tilde{b}_{11}^{k, 20}-\tilde{b}_{11}^{k-1,02}\right), & b_{11}^{k-1,02}:=b_{10}^{k, 20}+\frac{1}{2}\left(\tilde{b}_{11}^{k-1,02}-\tilde{b}_{11}^{k, 20}\right)
\end{array}
$$


where the coefficients $\tilde{b}_{21}^{k, 00}, \tilde{b}_{12}^{k-1,00}, \tilde{b}_{11}^{k, 10}$ and $\tilde{b}_{11}^{k-1,01}$ are obtained by subdividing the cubic Hermite interpolate to the transversal derivatives at the endpoints into three parts:

$$
\begin{aligned}
\tilde{b}_{21}^{k, 00} & :=-\frac{4}{9} b_{01}^{k, 00}+\frac{4}{3} b_{11}^{k, 00}+\frac{1}{3} b_{21}^{k, 20}-\frac{2}{9} b_{31}^{k, 20}, \\
\tilde{b}_{12}^{k-1,00} & :=-\frac{4}{9} b_{10}^{k-1,00}+\frac{4}{3} b_{11}^{k-1,00}+\frac{1}{3} b_{12}^{k-1,02}-\frac{2}{9} b_{13}^{k-1,02}, \\
\tilde{b}_{11}^{k, 10} & :=-\frac{20}{27} b_{01}^{k, 00}+\frac{4}{3} b_{11}^{k, 00}+b_{21}^{k, 20}-\frac{16}{27} b_{31}^{k, 20} \\
\tilde{b}_{11}^{k-1,01} & :=-\frac{20}{27} b_{10}^{k-1,00}+\frac{4}{3} b_{11}^{k-1,00}+b_{12}^{k-1,02}-\frac{16}{27} b_{13}^{k-1,02} .
\end{aligned}
$$

The coefficients $\tilde{b}_{21}^{k, 10} \tilde{b}_{12}^{k-1,01}, \tilde{b}_{11}^{k, 20}$ and $\tilde{b}_{11}^{k-1,02}$ are defined analogously.
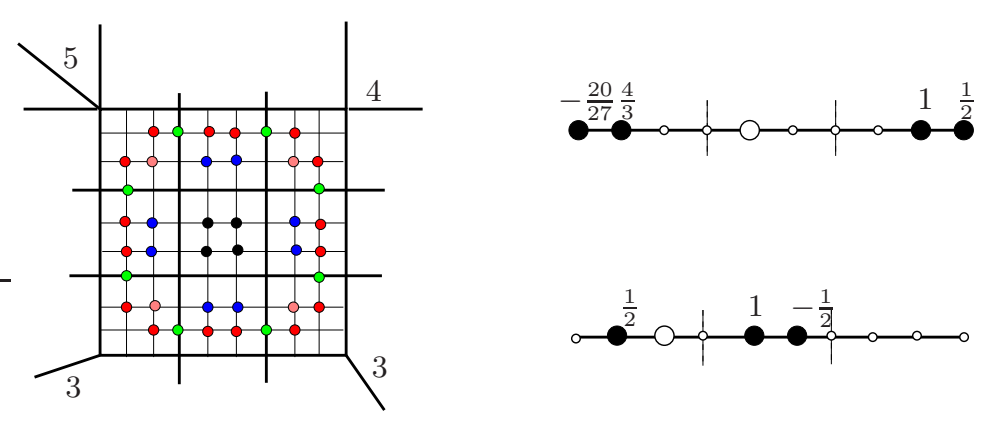

Fig. 3. left: Once the red BB-coefficients $b_{21}^{k, 00}, b_{11}^{k, 10}, b_{21}^{k, 10}, b_{11}^{k, 20}$ of the first interior layer are set, the green coefficients are $C^{1}$ averages of their two red neighbor points. Blue, pink and black coefficients are inner points computed by the rules on the right: (top) by subdivision and (bottom) so that the pieces join $C^{2}$ : the coefficient indicated by the large $\circ$ is a linear combination, with weights displayed, of the coefficients shown as

4. [macro-patch Interior] At the center (four black disks in Fig. 3, left) the coefficients are computed according to 3, right-top,

$b_{11}^{k, 11}:=\frac{\left(-\frac{20}{27} b_{01}^{k, 01}+\frac{4}{3} b_{11}^{k, 01}+b_{21}^{k, 21}+\frac{1}{2} b_{31}^{k, 21}\right)+\left(-\frac{20}{27} b_{10}^{k, 10}+\frac{4}{3} b_{11}^{k, 10}+b_{12}^{k, 12}+\frac{1}{2} b_{13}^{k, 12}\right)}{2}$,

and symmetrically for the other three corners. Coefficients marked in blue and pink are defined by the rules of Fig. 3 right-bottom and the remaining coefficients on the internal boundaries are the $C^{1}$ average of their neighbors, e.g. $b_{10}^{k, 11}:=$ $\left(b_{11}^{k, 11}+b_{21}^{k, 10}\right) / 2$ so that the patches join $C^{1}$ everywhere and $C^{2}$ with the central subpatch.

This completes the local construction of $C^{1} 3 \times 3$ macro-patches, one per input quad and so that neighbor macro-patches join $G^{1}$. Before we show examples, we discuss why we did not choose $m=2$. 


\section{Can $m=2$ provide a construction?}
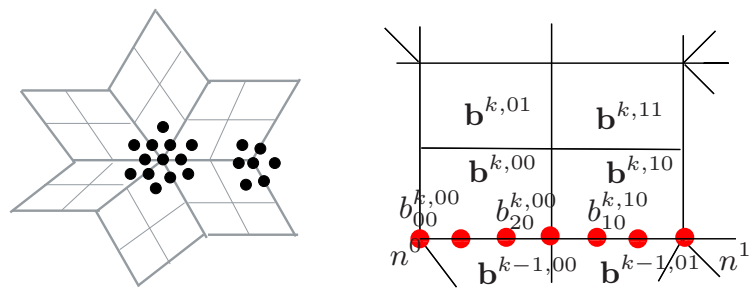

Fig. 4. left: Coefficients initialized according to (28). right: Indexing.

We show that an analogous construction is not possible for a $2 \times 2$ macropatch. Since the degree of $\partial_{2} \mathbf{b}^{k, \mu 0}(u, 0)$ and $\partial_{1} \mathbf{b}^{k-1,0 \mu}(0, u)$ is 3 , choosing $\alpha_{\mu}^{k}$ to be quadratic implies that $\partial_{1} \mathbf{b}^{k, \mu 0}(u, 0)$ must be linear, i.e. each boundary curve segment is piecewise quadratic. If both $n^{0}$ and $n^{1}$ are even and not 4 , then the vertex enclosure constraint (see Section 2) implies that the shared endpoint of the two quadratic segments is determined independently from both sides - so a local construction is not possible just as in the case $m=1$. We therefore choose $\alpha_{\mu}^{k}(u):=\lambda_{\mu}^{k}(1-u)+\lambda_{\mu+1}^{k} u, \mu=0,1$ with the unbiased choice (we do not prefer one sector over another)

$$
\lambda_{0}^{k}:=2 \cos \frac{2 \pi}{n^{0}}, \quad \lambda_{2}^{k}:=-2 \cos \frac{2 \pi}{n^{1}} .
$$

As in Section 3 (11), we enforce $(4)_{\mu=0}$ and $(7)_{\mu=1}$ of the eight $G^{1}$ continuity constraints by initializing position and tangents (black filled circles in Fig. 4 left) by subdividing $g$ :

$$
b_{00}^{k, 00}:=g_{00}^{k}, \quad b_{10}^{k, 00}:=\frac{g_{00}^{k}+g_{10}^{k}}{2}, \quad b_{11}^{k, 00}:=\frac{g_{00}^{k}+g_{10}^{k}+g_{01}^{k}+g_{11}^{k}}{4} .
$$

Lemma 1. If each macro-patch is parametrically $C^{1}, \alpha_{0}^{k}$ and $\alpha_{1}^{k}$ are linear with $\lambda_{0}^{k}$ and $\lambda_{2}^{k}$ unbiased then the $G^{1}$ constraints can only be enforced for all local initialization of $b_{00}^{k, 00}, b_{10}^{k, 00}, b_{11}^{k, 00}$ if $n^{0}=n^{1}$.

Proof. Due to the internal $C^{1}$ constraints, adding $(6)_{\mu=0}$ and $(5)_{\mu=1}$ and subtracting six times $(7)_{\mu=0}$ yields $3\left(v_{2}^{k, 0}+w_{2}^{k, 0}\right)+3\left(v_{1}^{k, 1}+w_{1}^{k, 1}\right)-6\left(v_{3}^{k, 0}+w_{3}^{k, 0}\right)=0$ and therefore the right hands sides satisfy

$$
\lambda_{0}^{k} u_{2}^{k, 0}+2 \lambda_{1}^{k} u_{1}^{k, 0}+2 \lambda_{1}^{k} u_{1}^{k, 1}+\lambda_{2}^{k} u_{0}^{k, 1}=6 \lambda_{1}^{k} u_{2}^{k, 0} .
$$

That is, for an internally $C^{1}$ macro-patch, $G^{1}$ constraints across the macropatch's boundary imply a constraint exclusively in terms of $u_{i}^{k, \mu}$, i.e. derivatives along the boundary! Since initialization fixes the local position, tangent and 
twist coefficients at each vertex, $(5)_{\mu=0}$ determines $b_{20}^{k, 00}$ and $(6)_{\mu=1}$ determines $b_{10}^{k, 10}$; and $C^{1}$ continuity determines $b_{30}^{k, 00}:=\left(b_{20}^{k, 00}+b_{10}^{k, 10}\right) / 2$. Thus all vectors of (29) are fixed and the remaining single free scalar $\lambda_{1}^{k}$ cannot always enforce (29). But if $n^{0}=n^{1}$ then $\lambda_{0}^{k}=-\lambda_{2}^{k}$ and $u_{2}^{k, 0}=u_{0}^{k, 1}$; and $\lambda_{1}^{k}=0$ solves (29).

\section{Conclusion}

Curvature distribution and highlight lines on the models of Fig. 5 illustrate the geometric soundness of the $m=3$ macro-patch construction. Choosing $\alpha_{1}^{k}$ and hence the middle boundary curve segment to be quadratic, avoids the PCCM shape problem which is due to $\alpha_{0}^{k}$ and hence the first segment being quadratic.

Conversely, Section 4 suggests that there is no obvious construction for $m=$ 2 ; whether a more complex Ansatz can yield a localized construction for $m=2$ remains the subject of research.

\section{References}

[CC78] E. Catmull and J. Clark. Recursively generated B-spline surfaces on arbitrary topological meshes. Computer Aided Design, 10:350-355, 1978.

[LS08] Charles Loop and Scott Schaefer. Approximating Catmull-Clark subdivision surfaces with bicubic patches. ACM Trans. Graph., 27(1):1-11, 2008.

[MYP08] A. Myles, Y. Yeo, and J. Peters. GPU conversion of quad meshes to smooth surfaces. In D. Manocha et al, editor, ACM SPM, pages 321-326, 2008.

$\left[\mathrm{NYM}^{+} 08\right]$ T. Ni, Y. Yeo, A. Myles, V. Goel, and J. Peters. GPU smoothing of quad meshes. In M. Spagnuolo et al, editor, IEEE SMI, pages 3-10, 2008.

[Pet00] Jörg Peters. Patching Catmull-Clark meshes. In K. Akeley, editor, ACM Siggraph, pages 255-258, 2000.

[Pet01] Jörg Peters. Modifications of PCCM. TR 001, Dept CISE, U Fl, 2001.

[Pet02] J. Peters. Geometric continuity. In Handbook of Computer Aided Geometric Design, pages 193-229. Elsevier, 2002.

\section{Appendix: linear parameterization and valence 4}

Lemma 2. If $k=4$ curves meet at a vertex without singularity and $\alpha_{i}^{k 0}$ is linear with $\lambda_{1}^{k}:=\ell \cos \frac{2 \pi}{n^{k}}$, for fixed scalar $\ell>0$ and valence $n^{k}$, then the $G^{1}$ constraints (3) can only be enforced if $n^{k}=n^{k+2}$ for $k=0,1$.

Proof. For $k=1,2,3,4, \lambda_{0}^{k}=0$ and therefore $\alpha_{i}^{k 0}:=\lambda_{1}^{k} u$. Equation $(5)_{\mu=0}$ then simplifies to

$$
3\left(b_{11}^{k, 00}+b_{11}^{k-1,00}\right)=6 b_{10}^{k, 00}+\lambda_{1}^{k} u_{0}^{k, 0}, k=1 \ldots 4 .
$$

Since $\sum_{k=1}^{4}(-1)^{k}\left(b_{11}^{k, 00}+b_{11}^{k-1,00}\right)=0$ and $\sum_{k=1}^{4}(-1)^{k} b_{00}^{k, 00}=0$,

$$
0=\sum_{k=1}^{4}(-1)^{k} 6\left(b_{10}^{k, 00}-b_{00}^{k, 00}\right)+\lambda_{1}^{k} u_{0}^{k, 0}=\sum_{k=1}^{4}(-1)^{k}\left(6+\lambda_{1}^{k}\right) u_{0}^{k, 0}
$$



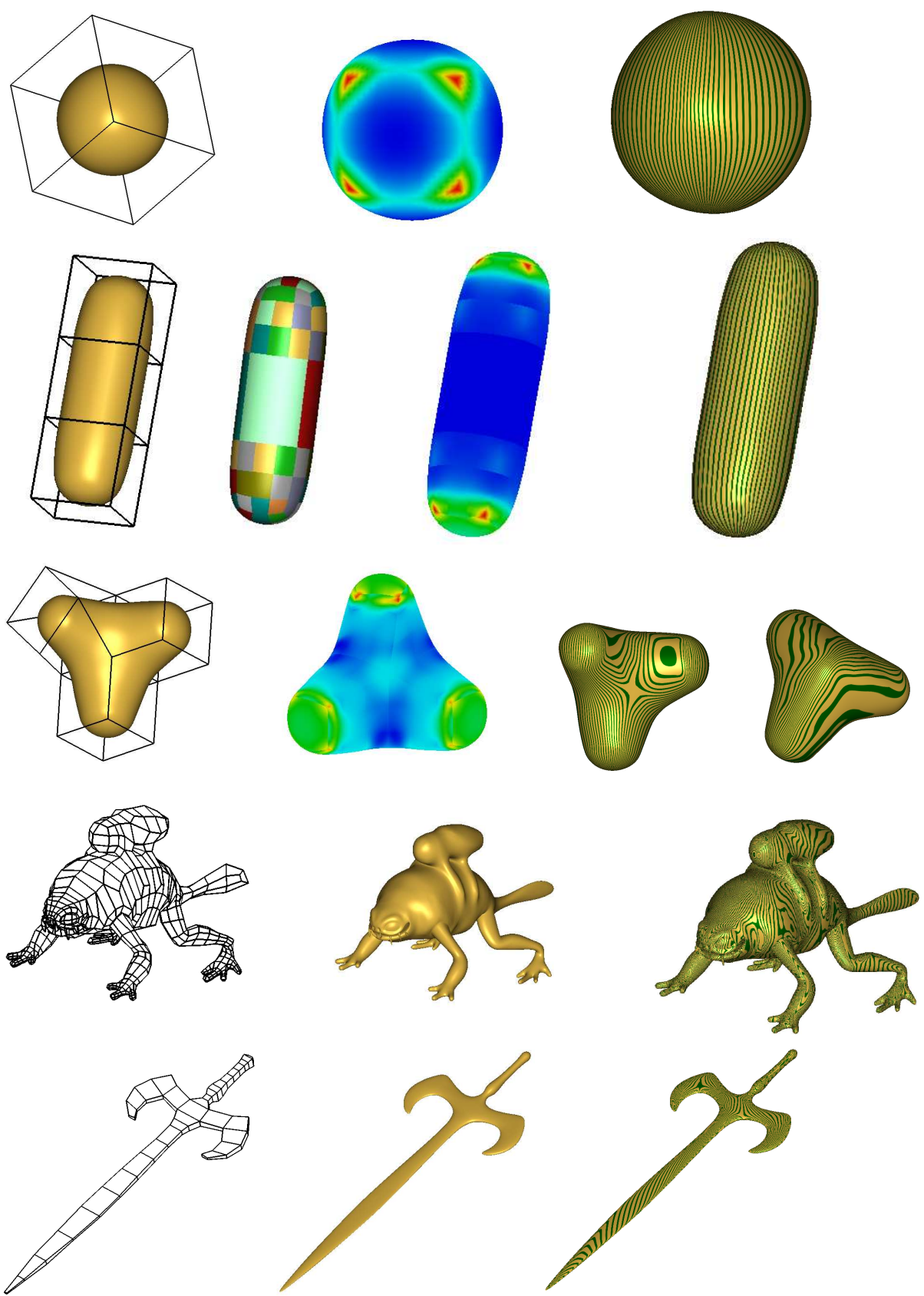

Fig. 5. $3 \times 3$ macro-patch construction. left: Quad mesh and surface; middle(top four): Gauss curvature distribution on the surface, right: Highlight lines. 
implying $\lambda_{1}^{k}=\lambda_{1}^{k+2}$ for $k=0,2$ since $u_{0}^{k, 0}=-u_{0}^{k+2,0}$. Therefore $n^{k}=n^{k+2}$ must hold.

Acknowledgement This work was supported by the National Science Foundation Grant 0728797. 